\title{
A Study of the Outcome of Critically Ill Elderly Patients in a Tertiary Care Hospital Using SOFA Score
}

\author{
Shivani Chopra ${ }^{1} \odot$, Sangeeta Pednekar ${ }^{2} \odot$, Niteen D Karnik ${ }^{3} \odot$, Charulata Londhe ${ }^{4} \odot$, Dharmendra Pandey ${ }^{5} \odot$
}

\begin{abstract}
Background: With a rapidly rising geriatric population, the magnitude of elderly patients requiring intensive care is a major cause of concern. Data on critically ill geriatric patients is scarce, especially in developing countries.

Aim and objective: The aim of the study is to identify the etiology, clinical profile, and outcome in elderly patients admitted to the intensive care unit (ICU) and to predict their survival using the sequential organ failure assessment (SOFA) score.

Materials and methods: A prospective observational study was performed over a period of 18 months with analysis of 100 patients admitted to the ICU, above the age of 60 years, with multi-organ dysfunction. The outcome of discharge or death was studied using the SOFA score on admission, on day 2 , and the delta SOFA score.

Results: In this study of 100 patients, $88 \%$ of patients were in the $60-70$ years age-group. The number of male and female patients was equal. Seventy percent of patients had comorbidities, of which hypertension was most common. The two most common etiologies were acute febrile illness and pneumonia. The use of mechanical ventilation, inotropic support, and serum creatinine has a significant association with the outcome. The SOFA score at admission did not have a significant association, but the score at 48 hours and delta SOFA score co-related with the outcome of the patients. Sixty-four patients got discharged; thus, there was a survival rate of $64 \%$.

Conclusion: The SOFA score at 48 hours is the most sensitive predictor of outcome, followed closely by the delta SOFA score, as compared to the SOFA score on admission, for critically ill elderly patients. There is a significant association of use of mechanical ventilation, inotropic support, and serum creatinine with the outcome.

Keywords: 48 hours, Critically ill adults, Elderly, Multi-organ dysfunction, Intensive care unit, SOFA score.

Indian Journal of Critical Care Medicine (2021): 10.5005/jp-journals-10071-23883
\end{abstract}

\section{INTRODUCTION}

With the demographic transition, there has been an expansion in the geriatric population. With the increasing life span, the proportion of patients belonging to the geriatric age-group is rising, thereby leading to an increase in the number of hospitalizations as well as demand for intensive care unit (ICU) facilities for the elderly patient population.

The elderly have multiple diseases and are complicated by social, psychological, and economic factors. In addition, these are superimposed by sensory and cognitive impairments. This cumulatively contributes to morbidity and mortality. Assessment of these factors can help in reducing the mortality by applying interventions in a timely manner.

In developing countries, the health-care resources are restricted. Therefore, it is necessary to have accurate prognostic markers justifying the transfer of geriatric patients to the ICU and guiding their treatment.

The sequential organ failure assessment (SOFA) score (Table 1) was initially named as sepsis-related organ failure assessment score. It was devised by the European Society of Intensive Care Medicine in 1994. It has been renamed as a sequential organ failure assessment (SOFA) score. Initially, it was used only in cases of sepsis but eventually came to be used extensively for nonsepsis-related patients. The use of SOFA score is to assess organ dysfunction over a period of time and evaluate morbidity in ICU patients. It is based on the degree of dysfunction of six organs on a scale of $0-4$ for each organ system. Delta SOFA is the change in the SOFA score between a baseline time and another defined time point.
${ }^{1-5}$ Department of Medicine, Lokmanya Tilak Municipal Medical
College, Mumbai, Maharashtra, India
Corresponding Author: Shivani Chopra, Department of Medicine,
Lokmanya Tilak Municipal Medical College, Mumbai, Maharashtra,
India, Phone: +91 9833550510, e-mail: chopralemons@gmail.com
How to cite this article: Chopra S, Pednekar S, Karnik ND, Londhe
C, Pandey D. A Study of the Outcome of Critically III Elderly
Patients in a Tertiary Care Hospital Using SOFA Score. Indian J Crit Care Med 2021;25(6):655-659.

Source of support: Nil

Conflict of interest: None

In this study, the SOFA score has been calculated on admission and at 48 hours in elderly patients in the ICU of a tertiary care hospital, and the outcome has been co-related.

\section{Materials and Methods}

This is an observational prospective study conducted in the inpatient department of a tertiary care hospital. This study was conducted over a period of 18 months, and patients were included by consecutive convenience sampling. Consecutive convenience sampling is subject to some errors like improper coverage and nonresponse of participants. All patients of age 60 years and above admitted to the medical ICU of our institute, with dysfunction of at least two organ systems, were considered eligible for this study. Valid informed consent was obtained from the patients or the

(c) Jaypee Brothers Medical Publishers. 2021 Open Access This article is distributed under the terms of the Creative Commons Attribution 4.0 International License (https://creativecommons.org/licenses/by-nc/4.0/), which permits unrestricted use, distribution, and non-commercial reproduction in any medium, provided you give appropriate credit to the original author(s) and the source, provide a link to the Creative Commons license, and indicate if changes were made. The Creative Commons Public Domain Dedication waiver (http://creativecommons.org/publicdomain/zero/1.0/) applies to the data made available in this article, unless otherwise stated. 
Table 1: The components of SOFA score

\begin{tabular}{|c|c|c|c|c|c|}
\hline Variables & 0 & 1 & 2 & 3 & 4 \\
\hline Respiratory $\left(\mathrm{PaO}_{2} / \mathrm{FiO}_{2}\right)(\mathrm{mm} \mathrm{Hg})$ & $>400$ & $<400$ & $<300$ & $<200$ & $<100$ \\
\hline Coagulation platelets $\times 10^{3} / \mu \mathrm{L}$ & $>150$ & $<150$ & $<100$ & $<50$ & $<20$ \\
\hline Liver serum bilirubin (mg/dL) & $<1.2$ & $1.2-1.9$ & $2.0-5.9$ & 6.0-11.9 & $>12.0$ \\
\hline Cardiovascular hypotension & No hypotension & $\begin{array}{l}\text { Mean arterial pres- } \\
\text { sure }<70 \mathrm{~mm} \mathrm{Hg}\end{array}$ & $\begin{array}{l}\text { Dopamine }<5 \text { or } \\
\text { dobutamine (any } \\
\text { dose) }\end{array}$ & $\begin{array}{l}\text { Dopamine }>5 \text {, Epi } \\
<0.1 \text {, or Norepi }<0.1\end{array}$ & $\begin{array}{l}\text { Dopamine }>15, \text { epi } \\
>0.1, \text { Norepi }>0.1\end{array}$ \\
\hline $\begin{array}{l}\text { Central nervous system } \\
\text { Glasgow coma scale }\end{array}$ & 15 & $13-14$ & $10-12$ & $6-9$ & $<6$ \\
\hline $\begin{array}{l}\text { Renal creatinine }(\mathrm{mg} / \mathrm{dL}) \text { or } \\
\text { Urine output ( } \mathrm{mL} / \text { day) }\end{array}$ & $<1.2$ & $1.2-1.9$ & $2.0-3.4$ & $\begin{array}{l}3.5-4.9 \\
<500\end{array}$ & $\begin{array}{l}>5.0 \\
<200\end{array}$ \\
\hline
\end{tabular}

Adrenergic agents are given for at least $1 \mathrm{hr}$ (doses given in $\mu \mathrm{g} / \mathrm{kg} / \mathrm{min}$ ); Epi, epinephrine; Norepi, norepinephrine

relative (in cases where the patient was not in full consciousness). Those who did not give consent were excluded from the study. Thus, of the 147 patients admitted, 100 qualified for the analysis. The study protocol has been approved by the Institutional Ethics Committee and Review Board. A case record form was used to obtain demographic, clinical, laboratory, and radiological data from the patients. Data on age, sex, comorbidity, and addictions were noted. The patients' vital parameters were noted, and general and systemic examinations were carried out. The worst values of the parameters used to obtain SOFA score were recorded on admission and at 48 hours. The parameters include partial pressure of arterial oxygen-to-fraction of inspired oxygen $\left(\mathrm{PaO}_{2} / \mathrm{FiO}_{2}\right)$, platelet count, serum bilirubin, serum creatinine, Glasgow Coma Scale (GCS), and blood pressure reading with the use of inotropic support. The values of the SOFA score and delta SOFA score were co-related with the outcome, which was either death or discharge.

\section{Statistical Analysis}

Data were entered on Microsoft Excel (Windows), and analysis was done using the Statistical Package for Social Sciences (SPSS) for Windows software (version 22.0). The mean and standard deviation (SD) for continuous variables, and frequencies and percentages were calculated for categorical variables. The Chi-square test was used for the analysis of the association between the categorical variables. The comparison of the mean of quantitative variables on day 0 and day 2 was done using the paired t-test. Visual representation of the analyzed data was done using bar charts and pie charts. The level of significance was set at 0.05 . The receiver operating characteristics curve was drawn to know the prediction of mortality by SOFA score.

\section{Results}

In this study of 100 patients more than 60 years of age, admitted to the medical ICU, the majority of the patients were in the age-group from 60 to 70 years (88\%). The average age of the patients was 65.51 years. There was an equal number of male and female patients in the study. Fifty-one percent of the patients in the study had a history of addictions. Seventy percent of patients had previous comorbidities on admission. Hypertension was seen in $50 \%$ of the patients (Table 2). The patients in our study had various diagnosis as depicted in Figure 1. The most common was acute febrile illness $(25 \%)$, followed by pneumonia (14\%). The others included cardiac failure, stroke, sepsis, urinary tract infection, chronic
Table 2: Distribution of study subjects according to the comorbidities $(N=100)$

\begin{tabular}{lcc}
\hline Comorbidities* $^{*}$ & No. & Percent \\
\hline CVA & 7 & 7.0 \\
TB & 9 & 9.0 \\
DM & 38 & 38.0 \\
HTN & 50 & 50.0 \\
CKD & 3 & 3.0 \\
RHD & 1 & 1.0 \\
\hline
\end{tabular}

CVA, cerebrovascular accident; TB, tuberculosis; DM, diabetes mellitus; HTN, hypertension; CKD, chronic kidney disease; RHD, rheumatic heart disease

obstructive airway disease, malignancy, chronic liver disease, acute gastroenteritis, poisoning, and Guillian-Barre syndrome.

The association of various organ systems with the outcome is shown in Table 3. The parameters with a significant association are the use of mechanical ventilation, serum creatinine on day 2 , and the sensorium as scored by the GCS.

The outcome of the study was co-related with the SOFA score on day 0 and day 2 , and delta SOFA score. The mean SOFA score on day 0 and day 2 and mean delta SOFA score were 8.12 and 7.76 and (-0.36), respectively (Table 4). The mean SOFA score on day 0 of the discharged patients was 7.73 and that of the expired patients was 8.81 ( $p$-value 0.056). On the contrary, the mean SOFA score on day 2 of the discharged patients was 5.22 and that of the expired patients was 12.28 ( $p$-value $<0.001$ ), while the mean delta SOFA scores of the discharged and expired patients were $(-2.52)$ and 3.47 , respectively ( $p$-value $<0.001$ ). Figure 2 shows the area under the curve (AUC) (range =0-1). The higher the AUC, the higher the prediction. The SOFA score on day 0 has an AUC of 0.606 , and AUC at day 2 is 0.957 , while the AUC for delta SOFA is 0.906 . Hence, SOFA at day 2 is a better predictor, followed by delta SOFA as compared to the SOFA score at day 0 in the study. Out of a total of 100 patients, 36 patients expired and the rest 64 were discharged. Thus, there was $36 \%$ mortality in the study.

\section{Discussion}

Many physicians are reluctant to admission for the elderly, the reasons being lack of resources, that ICU care can be deleterious, or that ICU care is not what the patient or family wants in their case. On the contrary, ICU physicians strongly support the admission 


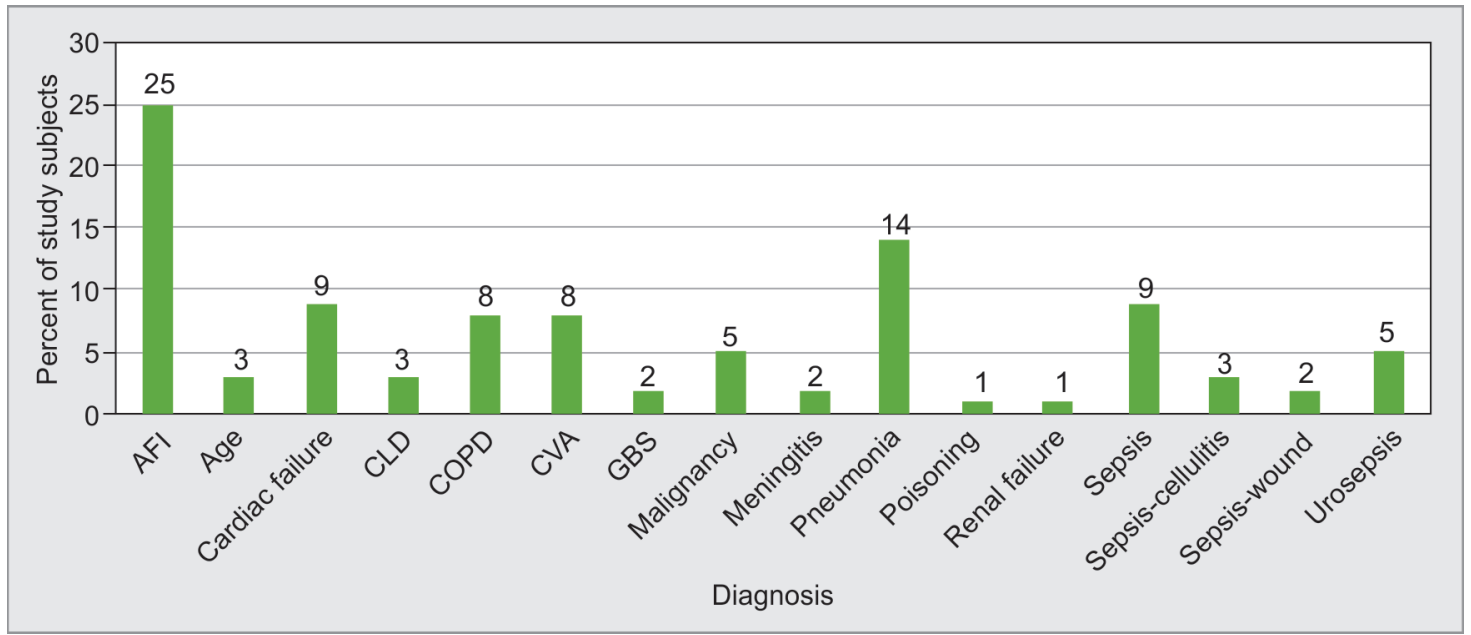

Fig. 1: Different etiologies of the study subjects

Table 3: Factors influencing outcome in the study $(N=100)$ with their significance ( $p$-value)

\begin{tabular}{|c|c|c|c|}
\hline Parameter & $\begin{array}{l}\text { Death mean } \\
\text { (SD) }\end{array}$ & $\begin{array}{l}\text { Discharged } \\
\text { mean (SD) }\end{array}$ & $p$-value \\
\hline Age (in years) & $66.89(6.81)$ & $64.73(6.48)$ & 0.121 \\
\hline $\mathrm{PaO}_{2} / \mathrm{FiO}_{2}$ day 0 & $247.28(94.63)$ & 300.09 (94.95) & $0.009^{*}$ \\
\hline $\mathrm{PaO}_{2} / \mathrm{FiO}_{2}$ day 2 & $173.64(72.05)$ & $350.69(83.48)$ & $<0.001^{*}$ \\
\hline HB day 0 & $10.61(2.28)$ & $10.34(2.65)$ & 0.604 \\
\hline HB day 2 & $10.34(2.10)$ & $10.07(2.11)$ & 0.543 \\
\hline WBC count day 0 & $14,740(10487)$ & $14,762(9598)$ & 0.991 \\
\hline WBC count day 2 & $13,836(8847)$ & $12,227(6661)$ & 0.307 \\
\hline Platelet count day 0 & $186.22(117.21)$ & 146.59 (122.6) & 0.118 \\
\hline Platelet count day 2 & $162.22(112.36)$ & $159.78(98.67)$ & 0.910 \\
\hline Bilirubin day 0 & $1.78(2.23)$ & $2.04(2.51)$ & 0.601 \\
\hline Bilirubin day 2 & $1.96(2.83)$ & $1.83(2.97)$ & 0.831 \\
\hline Creatinine day 0 & $3.03(2.00)$ & $2.95(2.04)$ & 0.839 \\
\hline Creatinine day 2 & $3.61(2.11)$ & $2.39(1.51)$ & $0.001^{*}$ \\
\hline GCS day 0 & $9.92(3.63)$ & $12.31(2.83)$ & $<0.001^{*}$ \\
\hline GCS day 2 & $6.72(2.53)$ & $13.42(2.36)$ & $<0.001^{*}$ \\
\hline SOFA day 0 & $8.81(2.79)$ & $7.73(2.57)$ & 0.056 \\
\hline SOFA day 2 & $12.28(2.46)$ & $5.22(2.84)$ & $<0.001^{*}$ \\
\hline \multirow[t]{2}{*}{ Delta SOFA } & $3.47(3.35)$ & $-2.52(2.66)$ & $<0.001^{*}$ \\
\hline & $n(\%)$ & $n(\%)$ & \\
\hline Inotropes day 0 & 12 & 21 & 0.958 \\
\hline No inotropes day 0 & 24 & 43 & \\
\hline Inotropes day 2 & 30 & 10 & $<0.001^{*}$ \\
\hline No inotropes day 2 & 6 & 54 & \\
\hline Addictions & 19 & 32 & 0.790 \\
\hline No addictions & 17 & 32 & \\
\hline Male & 20 & 30 & 0.405 \\
\hline Female & 16 & 34 & \\
\hline Mechanical ventilation & 36 & 34 & $<0.001^{*}$ \\
\hline
\end{tabular}

*Statistically significant; HB, hemoglobin; WBC, white blood cell; GCS, Glasgow coma scale; $\mathrm{paO}_{2}$, partial pressure of arterial oxygen; $\mathrm{FiO}_{2}$, fraction of inspired oxygen
Table 4: Distribution of study subjects according to the SOFA score $(N=100)$

\begin{tabular}{lllc}
\hline SOFA score & Day 0 & Day 2 & Delta SOFA \\
\hline Mean & 8.12 & 7.76 & $(-0.36)$ \\
SD & 2.69 & 4.34 & 4.10 \\
Range & $3-15$ & $1-18$ & $(-8)$ to 9 \\
\hline
\end{tabular}

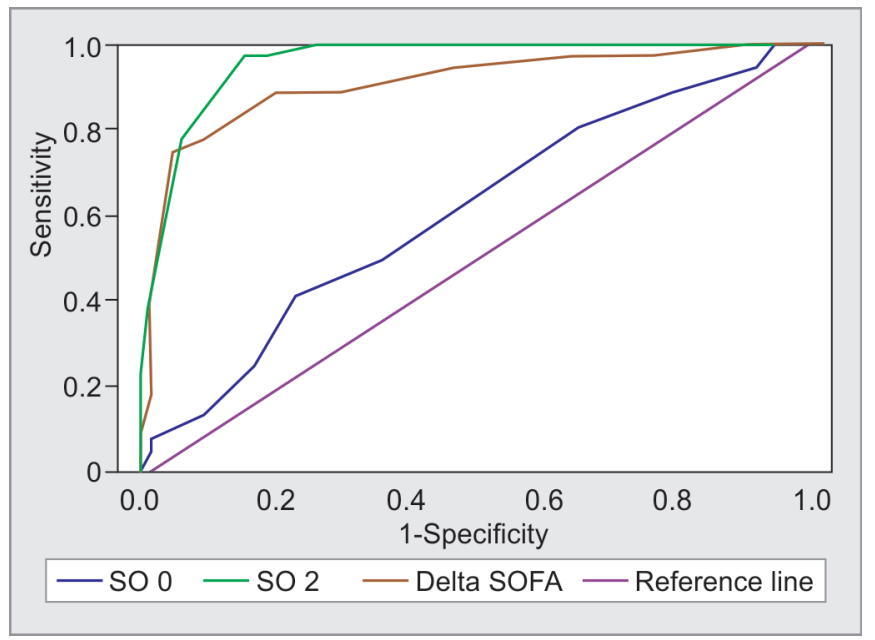

Fig. 2: Receiver operating characteristic curve showing AUC for SOFA score on admission, day 2 and delta SOFA score.

of elderly patients in selected cases. These discrepancies may be explained partially by the lack of validated criteria to accurately select the patients (of any age) who will benefit maximum from ICU admission. ${ }^{1}$ Age was an independent predictor of mortality, in the patients admitted with sepsis in the study by Martin et al. For this reason, despite the presence of clinical features indicating that intensive care is appropriate, ICU physicians are reluctant to consider ICU admission in elderly patients. ${ }^{2}$

Seventy percent of the study subjects had the presence of comorbidities. The most common comorbidity was hypertension, 
seen in $50 \%$ of the patients, while diabetes mellitus was the second most common accounting for $38 \%$ of the patients. Tuberculosis was seen affecting nine patients. Seven patients had a history of stroke. The other conditions included cardiac diseases like valvular heart disease and chronic kidney disease. Out of the 100 patients, 31 patients had more than one comorbidity. In a review by Guidet et al., comorbidities were associated with loss of physical independence, an increase in hospitalization rates, and increased mortality. Higher mortality in hospital and long-term mortality rates were seen in the ICU patients with comorbidities. ${ }^{3}$

In a study done by Gupta et al., of 84 elderly patients admitted to a tertiary care ICU, the most common etiology in critically ill elderly patients was stroke (23.8\%) followed by monsoon-related illness (16.67\%), pneumonia (11.9\%), malignancy (8.33\%), congestive cardiac failure (8.33\%), and Guillain-Barre syndrome and chronic obstructive pulmonary disease $(5.95 \%){ }^{4}$

\section{Association of Organ Systems with Outcome (Table 3) Respiratory}

Nearly $50.7 \%$ of the patients who required mechanical ventilation resulted in death, while all the patients who did not require mechanical ventilation got discharged. Thus, the association between mechanical ventilation and outcome is significant (with a $p$-value of $<0.001$ using the Chi-square test). The prognosis of elderly patients who developed acute lung injury and respiratory distress syndrome was studied in a prospective cohort study by Ely. The results showed that despite a similar rate of recovery from respiratory failure and achieving spontaneous respiration, among the elderly and young, the elderly had a prolonged ICU stay and a greater difficulty in weaning from mechanical ventilation. ${ }^{5}$

\section{Hemogram}

The mean hemoglobin and platelet values on both days did not have a significant association with the outcome of the patient in the study. In a study by Mukhopadhyay et al., it was found that each $1 \mathrm{~g} / \mathrm{dL}$ decrease in hemoglobin on ICU admission was associated with a $6 \%$ increase in the risk of mortality. This was accounted for by the fact that anemia is an indicator of poor general health and chronic illnesses. ${ }^{6}$ In a multicenter cohort study conducted on elderly patients with sepsis in the critical care unit, by Ignacio Martin-Looeches et al., thrombocytopenia was found to be associated independently with mortality in the elderly age-group. ${ }^{2}$

\section{Renal Function}

In this study, $84 \%$ of the patients with a serum creatinine value of less than $1.5 \mathrm{~g} / \mathrm{dL}$ got discharged, while $42 \%$ of the patients with a serum creatinine value of more than $1.5 \mathrm{~g} / \mathrm{dL}$ ended up with death. The mortality of the elderly critical patients with acute kidney injury in the study done by Santacruz et al. was more than $50 \%{ }^{7}$

\section{Neurologic}

On day 0 , out of the 17 patients with a poor GCS of less than $9,64.5 \%$ of the patients died. On day 2, out of the 27 patients with a poor GCS of less than $9,96.3 \%$ of patients died and all (100\%) of the patients with a GCS of more than 13 got discharged. The association of GCS with the outcome is significant. Thus, a poor sensorium denoted by a low GCS has been co-related to a worse outcome. Among different dysfunctional organ systems, the most significant correlation with the risk of mortality was due to neurological dysfunction, defined as the impaired level of consciousness, in the prospective observational cohort study by Vosylius et al. ${ }^{8}$

\section{Cardiovascular}

Thirty-three percent of patients required the use of inotropes on day 0 , and forty percent were on inotropic support on day 2 . The inotrope requirement on day 2 had a significant association with the outcome of the patients in the study. In a retrospective review of patients of $>85$ years of age admitted to the ICU, by Van Den Noortgate et al., the use of inotropes was an independent risk factor for in-hospital mortality. ${ }^{9}$ In another study by Mukopadhyay et al., there was an independent association of in-patient mortality with the use of mechanical ventilation and vasopressors. ${ }^{6}$

\section{SOFA SCORE}

SOFA score at day 2 is a better predictor of the outcome than the delta SOFA and SOFA score at day 0 in our study. In a systematic review by Minne et al. predicting the mortality risk in ICU patients, SOFA scores were found to have similar outcomes to the other organ failure scores. The combination of SOFA derivatives with APACHE II/III and SAPS II models is prognostically more accurate than either model alone. ${ }^{10}$ The delta SOFA was found to be a useful tool for risk stratification of patients in the emergency ward for admission to ICUs in the study by García-Gigorro et al. ${ }^{11}$ In the study by Qiao et al., the SOFA-initial, SOFA-48h, SOFA-96h, $\triangle$ SOFA, and SOFA-max scores were significantly lower in the patients who survived than in the patients who eventually succumbed. In this study, there was a higher degree of association of the mortality with the mean SOFA score and maximum SOFA score, as compared to the initial score. It was also found that a decreasing SOFA score during the first 48 hours was associated with a decrease in mortality rate from $50 \%$ to $27 \% .^{12}$ There was a significant association between mortality and SOFA score both at admission and at 48 hours, in a study on the correlation between SOFA score and outcome of elderly patients admitted to the ICU by Gupta et al. The mortality rate in their study was $78.4 \%$ in the elderly. ${ }^{4}$

\section{Limitations}

Ours is a single-center institutional study, and a multicenter study on similar lines in future would support and fortify the findings observed in our study.

\section{Conclusion}

The presence of comorbidities, rising serum creatinine, and use of inotropic support as well as mechanical ventilation adversely influence the prognosis of elderly patients in the ICU. The trend of the SOFA score from admission to 48 hours is a sensitive predictor of the outcome of critically ill elderly patients.

\section{HighLIGHTS}

- Seventy percent of elderly admitted to the medical ICU had at least one comorbidity.

- Acute febrile illness and pneumonia were the most common cause of illness. 
- The factors influencing the outcome include the use of mechanical ventilation, inotropic support, serum creatinine, and sensorium of the patient.

- SOFA score at 48 hours and delta SOFA score have a significant association with the outcome of the patient.

\section{OrCID}

Shivani Chopra 난 https:/orcid.org/0000-0002-5286-9723

Sangeeta Pednekar (1) https://orcid.org/0000-0002-4481-2946

Niteen D Karnik ㄴ https://orcid.org/0000-0002-5867-5911

Charulata Londhe (1) https://orcid.org/0000-0003-2003-7735

Dharmendra Pandey (1) https://orcid.org/0000-0003-2573-7212

\section{References}

1. Nguyen $Y-L$, Angus DC, Boumendil A, Guidet B. The challenge of admitting the very elderly to intensive care. Ann Intensive Care 2011;1(1):29. DOI: 10.1186/2110-5820-1-29.

2. Martin-Loeches I, Guia MC, Vallecoccia MS, Suarez D, Ibarz M, Irazabal $M$, et al. Risk factors for mortality in elderly and very elderly critically ill patients with sepsis: a prospective, observational, multicenter cohort study. Ann Intensive Care 2019;9(1):26. doi: 10.1186/s13613019-0495-x.

3. Guidet B, Vallet $H$, Boddaert J, de Lange DW, Morandi A, Leblanc G, et al. Caring for the critically ill patients over 80: a narrative review. Ann Intensive Care 2018;8:114. DOI: 10.1186/s13613-018-0458-7.

4. Gupta V, Karnik ND, Agrawal D. SOFA score and critically ill elderly patients and logistic organ dysfunction. J Assoc Physicians India
2017;65(July):60-63. Available at: https://pubmed.ncbi.nlm.nih. gov/28792169/

5. Ely EW, Wheeler AP, Thompson BT, Ancukiewicz M, Steinberg KP, Bernard GR. Recovery rate and prognosis in older persons who develop acute lung injury and the acute respiratory distress syndrome. Ann Intern Med 2002;136(1):25-36. Available at: https:// pubmed.ncbi.nlm.nih.gov/11777361/

6. Mukhopadhyay A, Tai BC, See KC, Ng WY, Lim TK, Onsiong S, et al. Risk factors for hospital and long-term mortality of critically ill elderly patients admitted to an intensive care unit. Biomed Res Int 2014;2014:960575. DOI: 10.1155/2014/960575.

7. Santacruz F, Barreto S, Mayor MM, Cabrera W, Breuer N. Mortality in elderly patients with acute renal failure. Ren Fail 1996;18(4):601-605. Available at: http://www.ncbi.nlm.nih.gov/pubmed/8875685

8. Vosylius S, Sipylaite J, Ivaskevicius J. Determinants of outcome in elderly patients admitted to the intensive care unit. Age Ageing 2005;34(2):157-162. DOI: 10.1093/ageing/afi037.

9. Van Den Noortgate N, Vogelaers D, Afschrift M, Colardyn F. Intensive care for very elderly patients: outcome and risk factors for in-hospital mortality. Age Ageing 1999;28(3):253-256. DOI: 10.1093/ ageing/28.3.253.

10. Minne L, Abu-Hanna A, de Jonge E. Evaluation of SOFA-based models for predicting mortality in the ICU: a systematic review. Crit Care 2008;12(6):R161. DOI: 10.1186/cc7160.

11. García-Gigorro R, Sáez-De La Fuente I, Marín Mateos H, AndrésEsteban EM, Sanchez-Izquierdo JA, Montejo-González JC. Utility of SOFA and $\triangle$-SOFA scores for predicting outcome in critically ill patients from the emergency department. Eur J Emerg Med 2018;25(6):387-393. DOI: 10.1097/MEJ.0000000000000472.

12. Qiao Q, Lu G, Li M, Shen Y, Xu D. Prediction of outcome in critically ill elderly patients using APACHE II and SOFA scores. J Int Med Res 2012;40(3):1114-1121. DOI: 10.1177/147323001204000331. 